\title{
Star formation at low rates - the impact of lacking massive stars on stellar feedback
}

\author{
Gerhard Hensler, Patrick Steyrleithner and Simone Recchi \\ University of Vienna, Dept. of Astrophysics, Tuerkenschanzstr. 17, 1180 Vienna, Austria \\ email: gerhard.hensler@univie.ac.at
}

\begin{abstract}
Due to their low masses dwarf galaxies experience low star-formation rates resulting in stellar cluster masses insufficient to fill the initial mass function (IMF) to the uppermost mass. Numerical simulations usually do not account for the completeness of the IMF, but treat a filed IMF by numbers, masses, and stellar feedback by fractions. To ensure that only entire stars are formed, we consider an IMF filled from the lower-mass regime and truncated where at least one entire massive star is formed.

By 3D simulations we investigate the effects of two possible IMFs on the evolution of dwarf galaxies: filled vs. truncated IMF. For the truncated IMF the star-formation self-regulation is suppressed, while the energy release by typeII supernovae is larger, both compared to the filled IMF. Moreover, the abundance ratios of particular elements yielded from massive and intermediate-mass stars differ significantly between the two IMF distributions.
\end{abstract}

Keywords. hydrodynamics, galaxies: star formation, galaxies: star clusters, galaxies: evolution

\section{Introduction}

Dwarf galaxies (DGs) are the most numerous type of galaxies in the universe. They have low surface brightnesses, low gravitational potentials, and mostly relatively low star-formation rates (SFRs). Due to their low gravitation they react more vigorously to external and internal processes, such as feedback by massive stars, and are therefore ideal objects to study galaxy evolution.

It is nowadays widely accepted that most stars are born in embedded clusters. Therefore, a mass distribution within each stellar cluster has to be assumed, the so-called initial mass function (IMF), originally defined by Salpeter (1955). The IMF is the most important distribution function in astrophysics, because stars of different masses affect the galactic evolution manifoldly, whereby strengths and timescales of their actions depend inversely on the mass. As part of the IMF, massive stars live most shortly and release most energy, mass, and heavy elements per stellar mass to their environment. Vice versa, the long-living, less active low-mass stars accumulate the stellar mass budget.

Since mostly SFRs cannot be derived directly from star counts in young star clusters, observational signatures are used to measure SFRs (see e.g. Kennicutt \& Evans 2012). For 300 star-forming galaxies in the local universe, Lee et al. (2009) compared SFRs derived from the $\mathrm{H} \alpha$ flux, a tracer for the most massive $\mathrm{O}$ stars, with those from the FUV, a tracer also including early-type B stars. For SFRs $\leqslant 10^{-2} M_{\odot} y r^{-1}$ they found $\mathrm{H} \alpha$-FUV flux ratios of one order of magnitude lower than expected and concluded that in DGs and low-surface brightness galaxies an IMF which is deficient of massive stars is consistent with their data. Interestingly, Larsen (2002) found a correlation between the maximum stellar cluster mass with SFR which, however, seems to be violated, if the SF is concentrated to a single region and produces a so-called super-star cluster as in starburst 
DGs. Whether massive-star formation happens deterministicly or stochasticly is under debate from observations (Andrews et al. 2014) and theoretically (see e.g. reviews by Kroupa (2014) and Krumholz (2014)). Moreover, the validity of $\mathrm{H} \alpha$ as SFR proxy must be questioned because of various reasons of stellar feedback. Both seems to be possible, to underestimate (Andrews et al. 2013) or to overestimate (Melekh et al. 2015) the SFR.

Numerical simulations of galaxy evolution nowadays include a lot of small-scale processes, like radiative gas cooling and stellar heating, star formation and chemical, energy and mass feedback, respectively, by supernovae (SNe) type Ia and II. Since it is computationally too demanding to resolve single stars on galactic scales, formed star clusters have to be treated as single stellar populations. To determine then the right stellar feedback, depending on stellar mass and lifetime, the IMF must be implemented properly. Since the IMF is thought to be invariant through large ranges of conditions (Kroupa 2001), an invariant IMF is used to describe individual star clusters numerically. When for simplicity a fully populated IMF is assumed as in most numerical models, at low SFRs the formed star-cluster mass cannot cover a complete IMF, but contains only fractions of massive stars. Due to this reason, a realistic IMF must be adapted either by a stochastic approach or by truncation at an uppermost mass bin that holds at least one star.

\section{The Simulations}

Since our study is dedicated to explore the effect of different IMF recipes on numerical models of DG evolution, we performed numerical experiments applying the adaptivemesh refinement code FLASH with its code version 3.3 (Fryxell et al. 2000) and extensions by us to the chemo-dynamical treatment (Hensler 2007). This includes a SF selfregulation recipe (Köppen, Theis, \& Hensler 1995), stellar feedback by massive stars' radiation and winds, gas cooling, and chemical enrichment. The models start with a purely gaseous disk, embedded into a DM halo. For the initial conditions an equilibrium configuration of a rotating gas disk is calculated, where the steady-state momentum equation for the gas component in a gravitational potential of gas and DM is solved (Vorobyov et al. 2012). For a detailed description see Steyleithner et al. (2016).

Our model DG consists of a DM halo mass of $M_{D M}=10^{10} M_{\odot}$ and a spin parameter of $\alpha=0.9$ so that the resulting gas mass is $M_{g}=1.4 \times 10^{8} M_{\odot}$ with a maximum rotation velocity of $30 \mathrm{~km} \mathrm{~s}^{-1}$. A radius $R_{\text {gal }}=9.5 \mathrm{kpc}$ is well defined by a minimum gas density of $\rho=10^{-27} \mathrm{~g} \mathrm{~cm}^{-3}$. Outside $R_{\text {gal }}$ the gas density is set to $10^{-30} \mathrm{~g} \mathrm{~cm}^{-3}$ and the temperature to $\mathrm{T}=10^{6} \mathrm{~K}$ to achieve pressure equilibrium with the galaxy. The DM mass within $R_{\text {gal }}$ then amounts to $M_{D M,<\text { Rgal }}=8.4 \times 10^{8} M_{\odot}$.

We simulate two DGs, with identical initial conditions: In one simulation all mass bins of a Kroupa IMF with an upper mass (independent on the cluster mass) of $120 M_{\odot}$ are filled, allowing the formation of fractions of massive stars. The other IMF is filled from low masses and truncated at an uppermost mass which cannot form a single star.

\section{The IMF-dependent issues}

The two different IMFs develop the following consequences for the DG evolution:

1. Because of the steeply inclining Lyman continuum flux $L y_{c}$-stellar mass power law with a power of 4-6 (Ploeckinger et al. 2015), even with massive-stars' mass fractions the filled IMF produces more total $L y_{c}$ energy. This leads to a stronger SF self-regulation so that the SFR is higher for the truncated IMF. 

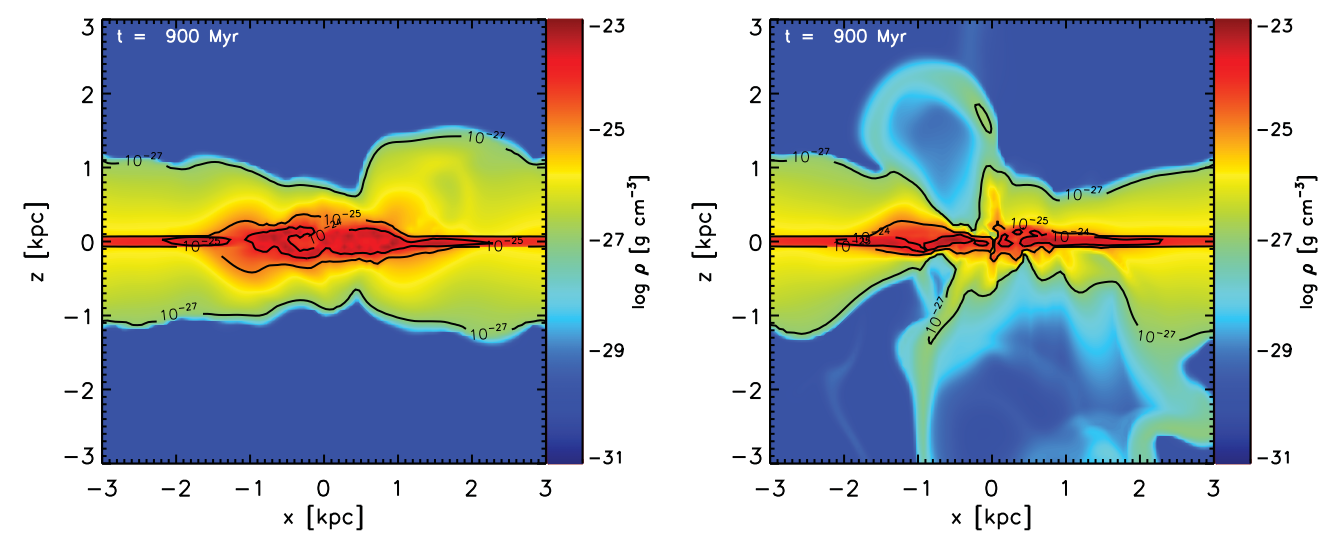

Figure 1. Edge-on slice through the filled IMF model (left) and truncated IMF model (right) at the same simulation time. The colour bar is given in logarithmic volume densities.

2. Because of the declining number of stars with mass according to the IMF, fractions of SNII explosive energies in the filled IMF produce less SNII energy than a few single explosions by the truncated IMF, but with the full $10^{51}$ ergs each.

3. Therefore, the truncated IMF exhibits a strong bipolar outflow in fig.1 (right).

4. The lack of massive stars results in lower yields of $\alpha$-elements with respect to the intermediate-mass stellar yields of e.g. C, Ba, and Fe from SNeIa what e.g. becomes discernible in dwarf spheriodals (Ploeckinger et al. 2015) and extremely metal-poor halo stars (Steyleithner et al. (2016)).

\section{Acknowledgements}

The authors are grateful to Sylvia Ploeckinger for her contributions and continuous discussions.

\section{References}

Andrews, J. E., Calzetti, D., Chandar, R., et al. 2013, ApJ, 767, 51

Andrews, J. E., Calzetti, D., Chandar, R., et al. 2014, ApJ, 793, 4

Fryxell, B., Olson, K., Ricker, P., et al. 2000, ApJS, 131, 273

Hensler, G. 2007, in eds. E. Ensellem et al., EAS Publ. Ser. No. , 7, p. 113

Kennicutt, R. C. \& Evans, N. J. 2012, ARA\& $A$, 50, 531

Köppen, J., Theis, C., \& Hensler, G. 1995, A\& $A$, 296, 99

Kroupa, P. 2001, MNRAS, 322, 231

Kroupa, P. 2014, Astrophys. and Space Sci. Proc., 36, 335

Krumholz, M. R. 2014, Phys. Rep., 539, 49

Larsen, S. S. 2002, AJ, 124, 1393

Lee, J. C., Elmegreen, B. G., Kennicutt, R. C., et al. 2009, ApJ, 774, 51

Melekh, B., Recchi, S., Hensler, G., \& Buhajenko, O. 2015, MNRAS, 450, 111

Ploeckinger, S., Hensler, G., Recchi, S., et al. 2014, MNRAS, 437, 3980

Ploeckinger, S., Recchi, S., Hensler, G., \& Kroupa, P. 2015, MNRAS, 447, 2512

Salpeter, E. E. 1955, ApJ, 121, 161

Steyrleiztner, P., Hensler, G., Recchi, S., \& Ploeckinger, S. 2016, MNRAS, submitted

Vorobyov, E. I., Recchi, S., \& Hensler, G. 2012, A\& A, 543, A129 